\title{
ADSORPTION PROPERTIES OF ACIDIFIED ACTIVATED CARBON FOR REMOVING IMPURITIES FROM PHENOL
}

\author{
Xuying Liu, Hao Zhang, Lili Lian, Xiyue Wang, Wenxiu Gao, Bo Zhu, Yanan Guan,Yang Xu, Dawei Lou* \\ Department of Analytical Chemistry, Jilin Institute of chemical Technology, 45 Chengde Street, Jilin 132022, PR China. \\ *Corresponding author email: $\underline{\text { dwlou@hotmail.com }}$
}

This is an open access article distributed under the Creative Commons Attribution License, which permits unrestricted use, distribution, and reproduction in any medium, provided the original work is properly cited.

\section{ARTICLE DETAILS}

\section{Article History:}

Received 26 June 2018 Accepted 2 July 2018

Available online 1 August 2018

\section{ABSTRACT}

2,4-diphenyl-4-methyl-1-pentene is a main impurity in phenol. In this paper, acidified activated carbon with sulfuric acid were used to remove the impurity from phenol. Combined with solid-phase microextraction (SPME) and gas chromatography-mass spectrometry (GC-MS), the adsorption capacity and mechanism of acidified activated carbon were analyzed. Unacidified and sulfated activated carbon was characterized by scanning electron microscopy (SEM). The adsorption mechanism of 2,4-diphenyl-4-methyl-1-pentene on sulfuric acid acidified activated carbon was investigated. The acidified activated carbon of sulfuric acid has much better adsorption capacity than the unacidified activated carbon on 2,4-diphenyl-4-methyl-1-pentene. The sulfuric acid acidified activated carbon has no obvious adsorption effectiveness on mesityl oxide and 2-methylbenzofuran. The optimum adsorption time of sulfuric acid acidified activated carbon for 2,4-diphenyl-4-methyl-1-pentene was $1 \mathrm{~h}$, the removal rate was $82 \%$. The Freundlich isothermal adsorption model can describe the adsorption behavior of 2,4-diphenyl-4-methyl-1-pentene on sulfuric acid acidified activated carbon. The adsorption process conforms to the pseudo-second-order kinetics model and is a chemical adsorption. The sulfuric acid acidified activated carbon can remove 2,4-diphenyl-4-methyl-1-pentene form phenol very well, which provide a theoretical basis and technical support for the phenol industry.

\section{KEYWORDS}

Acidified activated carbon, 2,4-diphenyl-4-methyl-1-pentene, adsorption capacity, adsorption mechanism. developed pore structure, abundant surface chemical groups, sufficient raw materials, high safety, and easy regeneration [13-15]. The surface chemical groups of activated carbon can be controlled by activating chemical processes or chemical post-treatment methods. That is, different chemical reagents are used to treat activated carbon, change the chemical or physical structure of the surface, and enhance the adsorption characteristics [16]. Because different adsorbates have different requirements for activated carbon. In order to achieve adsorption requirements for different adsorbates, activated carbon is usually subjected to targeted treatment to obtain desired physical and chemical properties. Therefore, because phenol is acidic, this article uses 50\% sulfuric acid to treat activated carbon. Combined with solid-phase microextraction (SPME) and gas chromatography-mass spectrometry (GC-MS) technology to explore the adsorption capacity of acidified activated carbon for organic impurities $[17,18]$. Further examine the adsorption mechanism of sulfuric acid acidified activated carbon to 2,4diphenyl-4-methyl-1-pentene, provides an effective method and relevant basis for the purification of phenol by enterprises.

\section{EXPERIMENTAL}

\subsection{Reagents}

The activated carbon were obtained from Tianjin Damao Chemical Reagent Factory (Tianjin, China). The phenols were obtained from Sinopec Jilin Dyeing Factory (Jilin, China). Methanol, 2-methylbenzofuran, and mesityl oxide were chromatographic purity. 2-methylbenzofuran was purchased from Sigma-Aldrich (Shanghai, China). Mesityl oxide was purchased from Alfa Aesar Chemical (Shanghai, China). Methanol was purchased from Tianjin Yongda Chemical Reagent Development Center (Tianjin, China). 2,4-diphenyl-4-methyl-1-pentene was purchased from Tokyo Chemical Industry Co., Ltd. (Tokyo, Japan). The ultrapure water was 
purified by a Milli-Q system (Millipore, Redford, MA, USA).

\subsection{Instrument}

Extraction was performed on an SPME extraction head (Supelco, PDMS, $100 \mu \mathrm{l}, 3 \mathrm{pk}, \mathrm{USA})$. The analysis was performed on an M7 single quadrupole GC-MS (Beijing Purkinje General Instrument Co., Ltd., Beijing, China). The inlet temperature was $240^{\circ} \mathrm{C}$. The reaction conditions were as follows: Program warming, the initial temperature was $50{ }^{\circ} \mathrm{C}$, kept for 0 min; the temperature was raised to $100{ }^{\circ} \mathrm{C}$ at $8{ }^{\circ} \mathrm{C} / \mathrm{min}$; the temperature was then raised to $200{ }^{\circ} \mathrm{C}$ at $10{ }^{\circ} \mathrm{C} / \mathrm{min}$ and next the temperature was raised to $290{ }^{\circ} \mathrm{C}$ at $15{ }^{\circ} \mathrm{C} / \mathrm{min}$ and kept for $5 \mathrm{~min}$. Pulsed split injection, with split ratio $10: 1$. The temperature of the ion source was $230^{\circ} \mathrm{C}$, and the temperature of the transmission line was $250^{\circ} \mathrm{C}$. SPME extraction was carried out on the DF-101S collector thermostatic heating magnetic stirrer (Gongyi Yuhua Instrument Co., Ltd., Henan, China). The static adsorption experiments were performed using a WE-1 water bath oscillator (Tianjin Uno Instruments Co., Ltd., Tianjin, China). The scanning electron microscopy (SEM) experiments were performed on a JSM-6490LV SEM (JEOL, Japan).

\subsection{Acidification of activated carbon}

For enhance the adsorption capacity of activated carbon, about $50 \mathrm{~g}$ activated carbon is placed in a beaker. The prepared sulfuric acid solution of $50 \%$ is put into the beaker. After mixing and treat for $48 \mathrm{~h}$, the sulfuric acid solution in the beaker to pour out as much as possible. The activated carbon after the sulfuric acid acidification was cleaned by the distilled water. Until the water poured out after cleaning was neutral. The cleaned activated carbon was placed in a drying oven at $110^{\circ} \mathrm{C}$ and dried for $12 \mathrm{~h}$. Cool to room temperature.

\subsection{Activated carbon static adsorption experiment}

\subsubsection{Adsorption capacity comparison experiment}

Firstly, 2,4-diphenyl-4-methyl-1-pentene phenol sample $2.5 \mathrm{~g}$ with concentration of $1 \mathrm{ppm}$ was added to $0.3,0.5,0.7,1.0,1.2$, and $1.5 \mathrm{~g}$ of untreated activated carbon. Adsorption $2 \mathrm{~h}$ in a water bath at a temperature of $50^{\circ} \mathrm{C}$. Then take out the sample and put it into the sample tube, test sample the next day. Then take $50 \%$ sulfuric acid acidified activated carbon with the same quality as above. Equal amount of 2,4diphenyl-4-methyl-1-pentene phenol sample was added. Adsorption $2 \mathrm{~h}$ in a water bath at a temperature of $50^{\circ} \mathrm{C}$. Take out the sample retain to the second day detection.

\subsubsection{Acidified activated carbon adsorbs different impurities}

In this work, $1 \mathrm{ppm}$ of 2,4-diphenyl-4-methyl-1-pentene, 2methylbenzofuran, and mesityl oxide phenol sample $2.5 \mathrm{~g}$ was added to $0.3,0.5,0.7,1.0,1.2$, and $1.5 \mathrm{~g}$ of sulfuric acid acidified activated carbon, respectively. Adsorption $2 \mathrm{~h}$ in a water bath at a temperature of $50{ }^{\circ} \mathrm{C}$, and the sample was taken out in a sample tube and detected the next day.

\subsection{Adsorption time}

Firstly, 5 parts of $0.5 \mathrm{~g}$ sulfuric acid acidified activated carbon into five 40 $\mathrm{mL}$ brown vials. Then $2.5 \mathrm{~g} 25 \mathrm{ppm}$ of 2,4-diphenyl-4-methyl-1-pentene phenol sample was added to the vials, Take another $2.5 \mathrm{~g}$ of 2,4-diphenyl4-methyl-1-pentene phenol sample as control. numbered 1-6, placed in a $50{ }^{\circ} \mathrm{C}$ water bath thermostat oscillator, according to the time gradient adsorptions of $0.5,1,2,3$ and $4 \mathrm{~h}$. Finally the samples were take out one by one and placed in a sample tube. The next day test.

\subsection{Adsorption process}

Take 5 parts of 0.5 g sulfuric acid acidified activated carbon. $2.5 \mathrm{~g}$ of 2,4diphenyl-4-methyl-1-pentene phenol sample at a concentration of 14,16, 18,20 and $22 \mathrm{ppm}$ were added to activated carbon, respectively. Adsorption $1 \mathrm{~h}$ in a water bath at a temperature of $50^{\circ} \mathrm{C}$. The samples were take out one by one and placed in a sample tube and detected the next day.

\section{RESULTS AND DISCUSSION}

\subsection{Activated carbon characterization}

SEM has been a primary tool for characterizing the surface morphology and fundamental physical properties of the adsorbent surface. SEM of untreated activated carbon and activated carbon was treated sulfuric acid are shown in Figure 1. It can be seen from Figure 1(A), has considerable numbers of pores on the surface of activated carbon. Compared with Figure 1 (A), As shown from the Figure 1(B), the activated carbon surface has more pores and larger pore size. This is due to the fact that during the acidification of activated carbon by sulfuric acid, closed pores are opened, fine pores are enlarged. This makes the average pore diameter of the activated carbon larger and increases the specific surface area of the activated carbon.

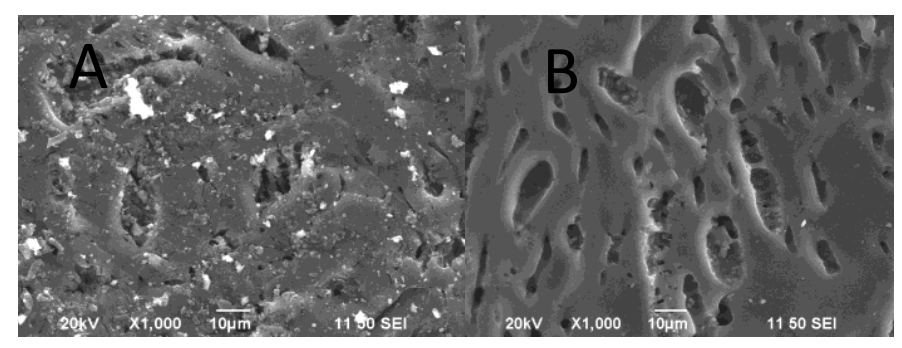

Figure 1: SEM image comparison of activated carbon (A. SEM image of untreated activated carbon; B. SEM image of sulfuric acid acidified activated carbon)

\subsection{Adsorption capacity of activated carbon}

The mass ratio of activated carbon under different acidifying conditions to phenol is $0.3: 2.5,0.5: 2.5,0.7: 2.5,1.0: 2.5,1.2: 2.5,1.5: 2.5$. At this mass ratio, the adsorption capacity of activated carbon for mesityl oxide, 2methylbenzofuran and 2,4-diphenyl-4-methyl-1-pentene in phenol was investigated. The mass of activated carbon and the adsorption rate are plotted in Figure 2. As shown in the figure 2(A), the untreated activated carbon has low adsorption capacity for 2,4-diphenyl-4-methyl-1-pentene. The adsorption effectiveness of the sulfuric acid acidified activated carbon for 2,4-diphenyl-4-methyl-1-pentene is significant. The mass ratio is $0.5: 2.5$ and adsorption rate can reach $80 \%$. Therefore, sulfuric acid acidified activated carbon has a good adsorption capacity for 2,4-diphenyl4-methyl-1-pentene. As seen from figure 2(B), The adsorption rates of mesityl oxide and 2-methylbenzofuran, with the increase of the mass of sulfuric acid acidified activated carbon are basically unchanged and the adsorption rate is lower. At the same time, compared with the adsorption capacity of sulfuric acid acidified activated carbon for mesityl oxide and 2 methylbenzofuran, sulfuric acid acidified activated carbon has a better adsorption capacity for 2,4-diphenyl-4-methyl-1-pentene. Therefore, sulfuric acid activated carbon can be used to remove 2,4-diphenyl-4methyl-1-pentene.
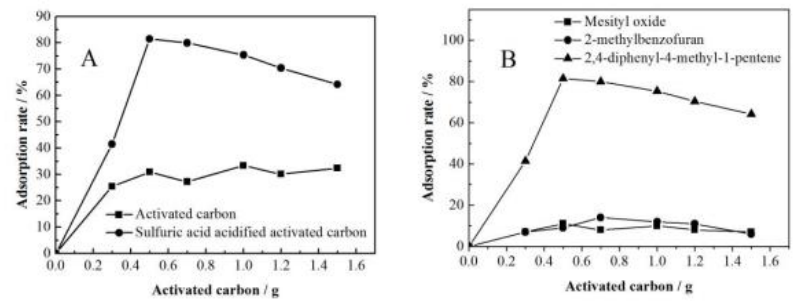

Figure 2: Adsorption effectiveness of mesityl oxide and 2methylbenzofuran, and 2,4-diphenyl-4-methyl-1-pentene by different acidified activated carbons (A. Adsorption capacity of 2,4-diphenyl-4methyl-1-pentene on activated carbon under different acidification conditions; B. Adsorption capacity of mesityl oxide and 2methylbenzofuran, and 2,4-diphenyl-4-methyl-1-pentene on sulfuric acid acidified activated carbon 


\subsection{Adsorption time}

The effect of adsorption time on the adsorption of 2,4-diphenyl-4-methyl1-pentene by sulfuric acid acidified activated carbon cannot be ignored. Adequate adsorption time can equilibrium the adsorption and improve the adsorption efficiency. As shown in the figure 3, the adsorption process show a very rapid rate in the beginning of adsorption, with the increase of time, the adsorption rate achieves the equilibrium after adsorption for $1 \mathrm{~h}$. Therefore, the best adsorption time was $1 \mathrm{~h}$.

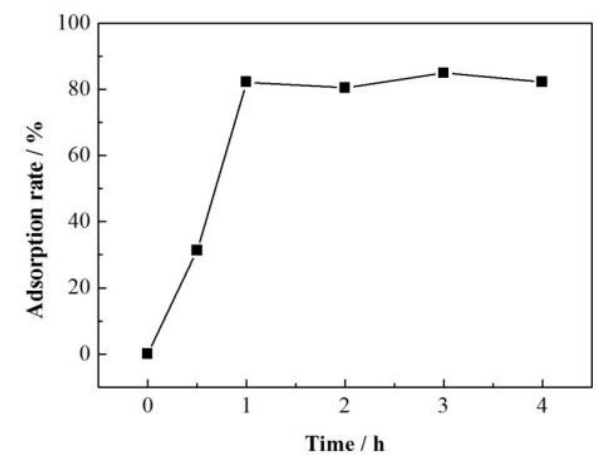

Figure 3: Effect of adsorption times on the adsorption of the 2,4diphenyl-4-methyl-1-pentene on sulfuric acid acidified activated carbon

\subsection{Adsorption isotherms}

The adsorption isotherms were fitted using the Langmuir equation and the Freundlich equation $[19,20]$. The Langmuir isothermal adsorption model describes that the adsorption is a monolayer adsorption process. The Freundlich isothermal adsorption model describes an multi-layer adsorption process on the adsorbent surface. These two equations are as follows:

Langmuir equation:

$$
\frac{C_{e}}{Q_{e}}=\frac{C_{e}}{Q_{m}}+\frac{1}{Q_{m} K_{L}}
$$

Freundlich equation:

$$
\ln \mathrm{Q}=\frac{1}{\mathrm{n}} \ln \mathrm{C}_{\mathrm{e}}+\ln \mathrm{K}
$$

In the equations, $Q_{e}$ represents the equilibrium adsorption capacity per unit weight of sulfuric acid acidified activated carbon, $C_{e}$ represents the equilibrium concentration, $\mathrm{K}_{\mathrm{L}}$ represents the Langmuir equilibrium adsorption constant, $\mathrm{Q}_{\mathrm{m}}$ represents the maximum adsorption capacity per unit mass of the sulfuric acid acidified activated carbon, $\mathrm{K}$ represents Freundlich equilibrium adsorption constant, and $\mathrm{n}$ represents the intensity coefficient.

From Eq. (1) and (2), the fitting results of the isothermal adsorption model fitting experimental data are shown in Figure 4, and the isothermal adsorption model parameters in Table 1 . The results show, the correlation coefficient of the Freundlich isothermal adsorption equation for 2,4diphenyl-4-methyl-1-pentene was 0.99 , which was larger than the correlation coefficient of the Langmuir isotherm equation of 0.96 . So the Freundlich equation can accurately describe the adsorption characteristics of 2,4-diphenyl-4-methyl-1-pentene on sulfuric acid acidified activated carbon, indicating that the adsorption is multi-layer.

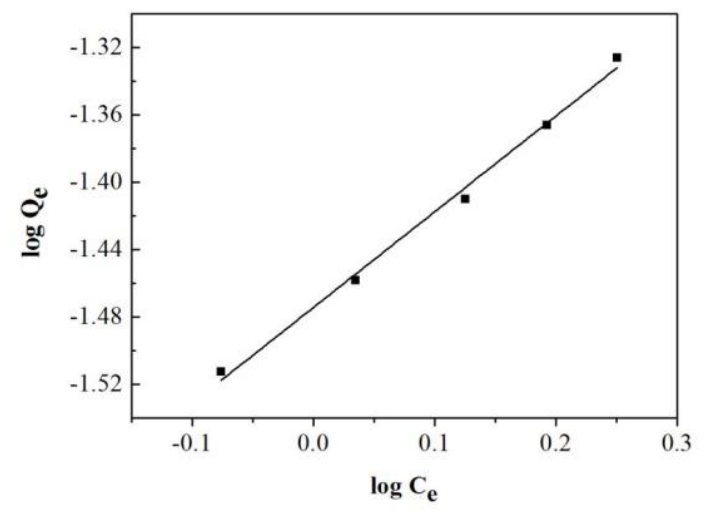

Figure 4: Freundlich isotherm model fitting results for 2,4-diphenyl-4methyl-1-pentene

Table 1: Langmuir and Freundlich isotherm model parameters for 2,4diphenyl-4-methyl-1-pentene

\begin{tabular}{|llllll|}
\hline Langmuir & \multicolumn{5}{c|}{ Freundlich } \\
\hline $\mathrm{Q}_{\mathrm{m}}(\mu \mathrm{g} / \mathrm{g})$ & $\mathrm{K}_{\mathrm{L}}$ & $\mathrm{R}^{2}$ & $1 / \mathrm{n}$ & $\mathrm{K}$ & $\mathrm{R}^{2}$ \\
0.0908 & $(\mathrm{~L} / \mathrm{mg})$ & & & & \\
& 0.5859 & 0.9645 & 0.5682 & 0.2289 & 0.9944 \\
\hline
\end{tabular}

\subsection{Adsorption kinetics}

The pseudo-first-order kinetics model and pseudo-second-order kinetics model were used to fit the adsorption times of 2,4-diphenyl-4-methyl-1pentene $[21,22]$. The correlation coefficient was used as the evaluation criterion. The pseudo-first-order kinetics model described the physical adsorption process; the pseudo-second-order kinetics adsorption model described the chemisorption process. These two kinetics expressions are as follows:

Pseudo-first-order kinetics model:

$$
\ln \left(\mathrm{Q}_{\mathrm{e}}-\mathrm{Q}_{\mathrm{t}}\right)=\ln \mathrm{Q}_{\mathrm{e}}-\mathrm{K}_{1} \mathrm{t}
$$

Pseudo-second-order kinetics model:

$$
\frac{\mathrm{t}}{\mathrm{Q}_{\mathrm{t}}}=\frac{1}{\mathrm{~K}_{2} \mathrm{Q}_{\mathrm{e}}^{2}}+\frac{\mathrm{t}}{\mathrm{Q}_{\mathrm{e}}}
$$

Where $Q_{e}$ is the amount of adsorption at equilibrium ( $\left.\mathrm{mg} \mathrm{g}^{-1}\right), Q_{t}$ is the amount of adsorption at time $\mathrm{t}\left(\mathrm{mg} \mathrm{g}^{-1}\right)$, $\mathrm{t}$ is the adsorption time $(\mathrm{h}), \mathrm{K}_{1}$ is the pseudo-first-order kinetics model adsorption equilibrium rate constant $\left(\mathrm{h}^{-1}\right)$, and $\mathrm{K}_{2}$ is the pseudo-second-order model adsorption equilibrium rate constant $\left(\mathrm{g} \mathrm{mg}^{-1} \mathrm{~h}^{-1}\right)$.

A pseudo-first-order kinetics model and pseudo-second-order kinetics model were used (Eq. 3 and 4) for fitting 2,4-diphenyl-4-methyl-1-pentene experimental data. Pseudo-second-order kinetics fitting results are shown in Figure 5, and the fitting parameters in Table 2. The results show that the correlation coefficient 0.99 of the pseudo-second-order kinetics model of 2,4-diphenyl-4-methyl-1-pentene is greater than the correlation coefficient 0.62 of the pseudo-first-order kinetics model. So the pseudosecond-order kinetics model can better describe the adsorption process of 2,4-diphenyl-4-methyl-1-pentene on sulfuric acid acidified activated carbon. Therefore, the reaction process is a chemical adsorption process. 


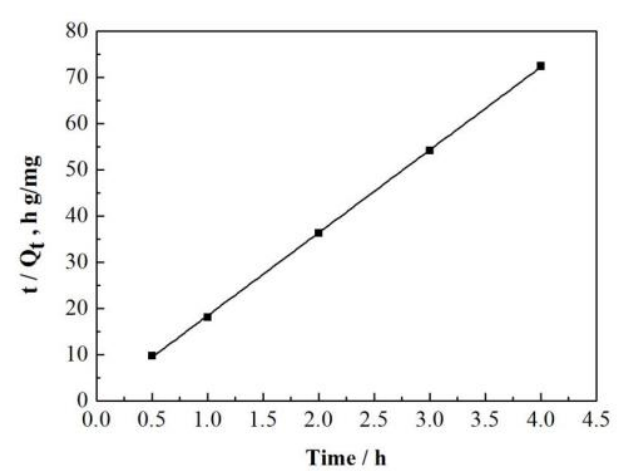

Figure 5: Fitting results of pseudo-second-order kinetics model to adsorption time on 2,4-diphenyl-4-methyl-1-pentene

Table 2: 2,4-diphenyl-4-methyl-1-pentene of pseudo-first-order and pseudo-second-order kinetics parameters

\begin{tabular}{|llllll|}
\hline \multicolumn{7}{|c|}{ Pseudo-first } & \multicolumn{5}{l|}{ Pseudo-second } \\
\hline $\mathrm{Q}_{\mathrm{e}, \mathrm{cal}}$ & $\mathrm{K}_{1} \quad\left(\mathrm{~h}^{-}\right.$ & $\mathrm{R}^{2}$ & $\mathrm{Q}_{\mathrm{e}, \text { cal }}$ & $\mathrm{K}_{2}$ & $\mathrm{R}^{2}$ \\
$(\mathrm{mg} / \mathrm{g})$ & $\left.{ }^{1}\right)$ & & $(\mathrm{mg} / \mathrm{g})$ & $(\mathrm{g} /(\mathrm{mg} \cdot \mathrm{h}))$ & \\
0.1513 & 2.425 & 0.623 & 0.0556 & 688.6774 & 0.999 \\
& 9 & 3 & & & 9 \\
\hline
\end{tabular}

\section{CONCLUSIONS}

In summary, we successfully found a method to remove impurities from phenol by using activated carbon as adsorbent. Sulfuric acid acidified activated carbon has good adsorption capacity for 2,4-diphenyl-4-methyl1-pentene, and the removal rate is $82 \%$. Both the Freundlich model and the pseudo-second-order kinetics model can well describe the behavior of 2,4-diphenyl-4-methyl-1-pentene in activated carbon. Acidified activated carbon can be used to remove 2,4-diphenyl-4-methyl-1-pentene. This provides a theoretical basis and a new approach for phenol companies purification of phenol.

\section{ACKNOWLEDGMENTS}

This work was supported by the National Natural Science Foundation of China [grant numbers 21375046, 2160505]; project of Science and Techn ology Development of Jilin Province (grant number20140203013GX). The financial support from the Key Laboratory of Fine Chemicals of Jilin $P$ rovince is also acknowledged.The authors have declared no conflict of int erest.

\section{REFERENCES}

[1] Bertran, M.O., Galvanin, S., Mattei, M. 2013. Production of Phenol from Cumene - Systematic and Efficient Design Method. Aiche Meeting.

[2] Kaymakcioglu, N., Yagci, G., Simsek, A., Unlu, A., Tekin, O. F., Cetiner, S. 2005. Treatment of pilonidal sinus by phenol application and factors affecting the recurrence. Techniques in Coloproctology, 9 (1), 21.

[3] Olmez, A., Kayaalp, C., Aydin, C. 2013. Treatment of pilonidal disease by combination of pit excision and phenol application. Techniques in Coloproctology, 17 (2), 201-206.

[4] Wang, H.J., Sheng-Yan, W.U. 2010. Measures of phenol consumption reducing in salicylic acid production. Technology \& Development of Chemical Industry.

[5] Hugo, W.B. 1978. Phenols: a review of their history and development as antimicrobial agents. Microbios, 23 (92), 83.

[6] Knifton, J.F. 1989. Method for production of phenol/acetone from cumene hydroperoxide. US, US4870217.
[7] Zhang, Y., Wang, Y. 2002. Evaluation on the method of measuring the concentration of cumene hydroperoxide of the phenol-acetone unit. Petrochemical Industry Technology.

[8] Molinari, R., Poerio, T., Argurio, P. 2006. One-step production of phenol by selective oxidation of benzene in a biphasic system. Catalysis Today, 118 (1), 52-56.

[9] Denton, W.I., Doherty, H.G., Krieble, R.H. 1950. Production of phenol by direct oxidation of benzene. Industrial \& Engineering Chemistry, 42 (5), 777-786.

[10] Selvin, R., Hsu, H.L., Aneesh, P., Chen, S.H., Li, L.H. 2010. Preparation of acid-modified bentonite for selective decomposition of cumene hydroperoxide into phenol and acetone. Reaction Kinetics Mechanisms and Catalysis, 100 (1), 197-204.

[11] Fulmer, J.W., Vaish, N., Kumbhar, P., Oberholtzer, J.L., Mahalingam, J.R. 2003. Production and purification of phenol. US, US 6635789 B2.

[12] Tsypysheva, L.G., Mishchenko, L.Y., Portnova, T.V., Kruglov, E.A., Yamshanova, N. M. 1987. Identification of impurities in commercial phenol produced by the cumene process. J. Anal. Chem. USSR (Engl. Transl.); (United States), 42, 2 (2), 283-287.

[13] Lua, A.C., Yang, T. 2004. Effect of activation temperature on the textural and chemical properties of potassium hydroxide activated carbon prepared from pistachio-nut shell. Journal of Colloid \& Interface Science, 274 (2), 594.

[14] Wang, S., Lu, G.Q. 1998. Effects of acidic treatments on the pore and surface properties of ni catalyst supported on activated carbon. Carbon, 36 (3), 283-292.

[15] Yuan, Y., Chen, Z., Qin, H.L., Han, Z.W. 2014. The new technology of preparation activated carbon from lignite. Applied Mechanics \& Materials, 483, 42-46.

[16] Vivo-Vilches, J.F., Bailón-García, E., Pérez-Cadenas, A.F., CarrascoMarín, F., Maldonado-Hódar, F.J. 2014. Tailoring the surface chemistry and porosity of activated carbons: evidence of reorganization and mobility of oxygenated surface groups. Carbon, 68 (3), 520-530.

[17] Zhang, Z., Yang, M.J., Pawliszyn, J. 1994. Solid-phase microextraction a solvent-free alternative for sample preparation. Analytical Chemistry, 66 (17).

[18] Buchholz, K.D., Pawliszyn, J. 1994. Optimization of solid-phase microextraction conditions for determination of phenols. Analytical Chemistry, 66 (1), 160-167.

[19] Huang, Z., Wu, Q., Liu, S., Liu, T., Zhang, B. 2013. A novel biodegradable $\beta$-cyclodextrin-based hydrogel for the removal of heavy metal ions. Carbohydr Polym, 97 (2), 496-501.

[20] Bashir, M.J.K., Aziz, H.A., Yusoff, M.S., Adlan, M.N. 2010. Application of response surface methodology (rsm) for optimization of ammoniacal nitrogen removal from semi-aerobic landfill leachate using ion exchange resin. Desalination, 254 (1-3), 154-161.

[21] Gopal, N., Asaithambi, M., Sivakumar, P., Sivakumar, V. 2014. Adsorption studies of a direct dye using polyaniline coated activated carbon prepared from prosopis juliflora. Journal of Water Process Engineering, 2, 87-95.

[22] Huang, Z., Liu, S., Zhang, B., Xu, L., Hu, X. 2012. Equilibrium and kinetics studies on the absorption of cu(ii) from the aqueous phase using a $\beta$-cyclodextrin-based adsorbent. Carbohydrate Polymers, 88 (2), 609617. 